SVN SVN limive

\title{
MRI and glymphatic system
}

\author{
Quan Jiang ${ }^{\odot}$
}

To cite: Jiang Q. MRI and glymphatic system. Stroke and Vascular Neurology 2019;4: e000197. doi:10.1136/svn2018-000197

Received 19 0ctober 2018 Revised 22 January 2019 Accepted 20 February 2019 Published Online First 5 April 2019
Check for updates

(C) Author(s) (or their employer(s)) 2019. Re-use permitted under CC BY-NC. No commercial re-use. See rights and permissions. Published by BMJ.

Departments of Neurology, Henry Ford Hospital, Detroit, Michigan, USA

Correspondence to Dr Quan Jiang; qjiang1@hfhs.org

\section{ABSTRACT}

Glymphatic system is newly discovered pseudolymphatic system in brain and it plays an important role in the removal of interstitial metabolic waste products. We discuss and review the role of glymphatic system in neurological diseases, especially focus on the potential of MRI modelling and its associated challenges to obtain useful new information related to understand the glymphatic system dynamics, pathways, and provide quantitative maps for diagnosis, monitoring and prognosis of the disease. The non-invasive nature of MRI might more readily help with translation of glymphatic measurements from the laboratory to the clinic.

\section{INTRODUCTION OF GLYMPHATIC SYSTEM}

The traditional concept of cerebrospinal fluid (CSF) hydrodynamics is being challenged by recent publications. ${ }^{1-6}$ The traditional model of CSF believes that CSF is produced in the choroid plexus in four ventricles, then flows from the ventricles to the subarachnoid spaces, and from there flows out along spinal and cranial nerves, the arachnoid villi and the olfactory bulb. Recent investigations provide evidence showing that a newly identified system, the glymphatic system, exists in the brain which facilitates the exchange of interstitial fluid (ISF) and CSF, and provide a pathway of organised convective fluid flow to drive clearance of interstitial solutes with wastes from the brain. ${ }^{1-6}$ The glymphatic system consists of para-arterial CSF influx and paravenous efflux routes, which are dependent on the astrocytic aquaporin-4 (AQP4) water channel. ${ }^{1-5}$ As demonstrated in figure 1, the influx flow from subarachnoid CSF enters parenchymal through para-arterial pathways and then exchanges with ISF facilitated by AQP4, both are cleared together with associated solutes along paravenous pathways, from where it exits into head lymphatic vessels. ${ }^{3}$ Weller et al defined paravascular drainage pathways in the brain, and speculated about its influence on $A \beta$ clearance from the brain. ${ }^{78}$ Iliff $e t a l$ had demonstrated the greatest tracer accumulation was detected surrounding large-calibre draining veins. ${ }^{3}$ Louveau et al and Aspelund et al have recently discovered that the meninges of a lymphatic network drains the macromolecules and immune cells from the brain parenchyma. ${ }^{69}$

\section{GLYMPHATIC SYSTEM AND NEUROLOGICAL DISEASES}

The glymphatic system plays an important role in neurological diseases. ${ }^{1-5} 10$ Xie et al demonstrated that natural sleep or anaesthesia significantly enlarged the interstitial space which increased convective exchange of CSF with ISF and clearance rate of $\beta$-amyloid during sleep. ${ }^{1}$ Ageing strongly relates to many neurodegenerative diseases and is also associated with a slow waste clearance in the brain. ${ }^{11}$ CSF tracer clearance was gradually reduced from middle-aged (12-13 months) to old (18 months) brain compared with young (2-3 months) brain. ${ }^{11}$ In mouse Alzheimer's disease model, Peng et al found reduced clearance of $\beta$-amyloid and glymphatic influx which worsened with advanced age. ${ }^{10}$ Reduced waste clearance has also been demonstrated after traumatic brain injury, haemorrhage, hypertension, acute ischaemia and multiple microinfarction. ${ }^{5}{ }^{12-16}$ In diabetes mellitus (DM) animal model, tracer clearance from brain parenchyma was significantly reduced with diabetes, leading to accumulation of tracers in multiple brain regions which correlated with degree of cognitive decline. ${ }^{17}$ Although glymphatic system has exhibited its important role in neurological diseases, further investigation is necessary to develop glymphatic directed therapeutic strategies.

\section{MRI DETECTION AND MODELLING OF GLYMPHATIC SYSTEM}

Most previous studies of the glymphatic system have been performed with two-photon laser scanning microscopy, which is precise and excellent for a predetermined small paravascular space but not a suitable tool for whole brain study, especially deep brain tissues and also invasive. MRI overcomes the weak points of two-photon confocal microscopy and provides non-invasive whole brain dynamic real-time imaging of the glymphatic system. Iliff et al first employed MRI to monitor glymphatic function and demonstrated that MRI allowed the identification of glymphatic pathways, several influx nodes and some kinetic parameters to evaluate 


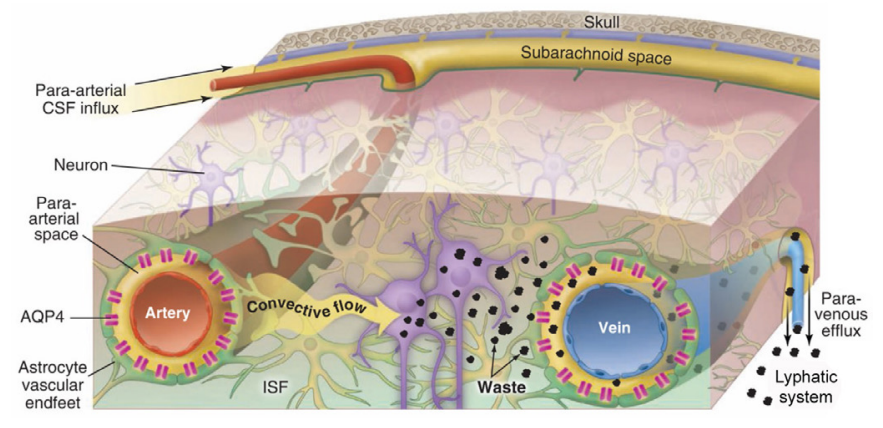

Figure 1 Influx, CSF-ISF exchange and efflux of glymphatic system: The influx flow of CSF into brain through the paraarterial pathways and then exchange with ISF. The mixed CSF and ISF with interstitial metabolic waste are flowed towards paravenous pathways, and then go to lymphatic system. AQP4 water channels in both arteries and veins play important role in reducing the resistance to CSF movement between paravascular spaces and the interstitium. Reproduced with permission from ${ }^{25}$. AQP4, astrocytic aquaporin-4; CSF, cerebrospinal fluid; ISF, interstitial fluid.

solute clearance in the brain. ${ }^{18}$ MRI has also exhibited its advantage to monitor whole brain pictures of slow waste clearance after subarachnoid haemorrhage and ischaemic stroke. ${ }^{131516}$ A recent study has demonstrated that compared with middle-aged non-DM animals, tracer clearance from brain parenchyma measured by both MRI and fluorescence imaging was significantly reduced with DM. ${ }^{17}$ MRI cluster analysis measurement of glymphatic system also exhibits a significant increase in paravascular space in the DM animals compared with control animals. Most interestingly, the MRI measures of tracer clearance, residual intensity and paravascular space are highly sensitive indices of pathological changes occurring early during the development of DM compared with functional behavioural tests and vascular dysfunction.
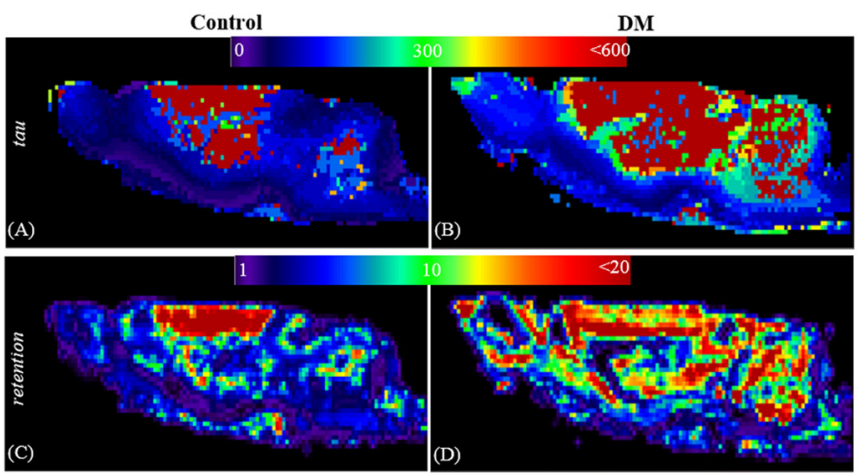

Figure 2 The maps of quantitative modelling the glymphatic system for control and DM animals. Figure 2 shows the visual comparison of the clearance time constant, tau (minutes, (A, $B)$ and the retention $(C, D)$ maps under control $(A, C)$ and $D M$ $(\mathrm{B}, \mathrm{D})$ conditions. Comparing with the control, DM animal exhibits longer clearance time constant (slower clearance of the tracer from the tissue) and high retention values (large fraction of the tracer remains in brain tissue). DM, diabetes mellitus.
Although MRI measurements of glymphatic system have exhibited a great potential for non-invasive evaluation of whole brain glymphatic functions, properly modelling this newly discovered system is still challenge. So far, few studies have been published for modelling glymphatic system. Ratner et $a l^{1920}$ identified the glymphatic pathways by using optimal mass transport to model the glymphatic flow vector field. Results from this study agreed with previous results of the glymphatic pathways. However, a weak point of this model is no quantitative measures of this system. Lee et al performed a very interesting study using kinetic modelling to quantify CSF-ISF exchange rates in supine, prone or lateral positions. This model exhibited the ability to differential the CSF-ISF exchange rates in three different postures and the quantitative maps derived from this model showed that glymphatic transport was most efficient in the lateral position compared with the supine or prone positions. ${ }^{21}$ One limitation for this model is its global input function which could produce more errors, especially for glymphatic system.

A newly published model using local input function has exhibited its advantage for improving the detecting sensitivity of glymphatic system. ${ }^{22}$ This model employed cluster analysis to determine the similar regions with the time signal curves from dynamic contrast agent MRI data and then derive local input function based on signal exchanges between regions. ${ }^{22}$ The results from this new model exhibit its better sensitivity to differential diabetic and control animals than previous two-compartment kinetic model and show increased clearance time and binding of tracers in DM compared with control animal brain (figure 2). Arterial input using global input function is a long-standing issue and the major source of error in cerebral permeability and perfusion measurements. Although model-based approach had tried to improve the delay and dispersion issues and to derive the local input function in permeability and perfusion measurements, ${ }^{23} 24$ directly measure local input function is still a challenge. Compared with vascular system, the errors caused global input delay and dispersion in glymphatic system are more severe due to much longer delay and tissue involved dispersion. The newly published model ${ }^{22}$ provides a better approach for more accurate evaluate glymphatic changes under neurological diseases.

Current MRI modelling of glymphatic system only considers a pressure-driven motion of bulk fluid referred to as 'advection'. The waste clearance could also involve diffusion although it will play much less role in the waste clearance. The further modelling of brain waste clearance could include both advection and diffusion to further reduce system errors.

\section{CONCLUSION}

Glymphatic system plays important role in neurological diseases. MRI could provide more information about glymphatic functions in whole brain study, especially 
deep brain tissues. Precisely modelling glymphatic system could obtain useful new information related to understand the glymphatic system dynamics, pathways, and provide quantitative maps for diagnosis, monitoring and prognosis of the disease.

Acknowledgements This work was supported by NIH grants RF1 AG057494, R01 NS108463, R01 NS097747 and R21 AG052735. I thank Li Zhang, Esmaeil DavoodiBojd, Guangliang Ding, Zhenggang Zhang and Michael Chopp for their contribution of the glymphatic studies in the Department of Neurology, Henry Ford Hospital.

Contributors $Q J$ is the sole author of the contribution.

Funding The authors have not declared a specific grant for this research from any funding agency in the public, commercial or not-for-profit sectors.

Competing interests None declared.

Patient consent for publication Not required.

Provenance and peer review Commissioned; externally peer reviewed.

Open access This is an open access article distributed in accordance with the Creative Commons Attribution Non Commercial (CC BY-NC 4.0) license, which permits others to distribute, remix, adapt, build upon this work non-commercially, and license their derivative works on different terms, provided the original work is properly cited, appropriate credit is given, any changes made indicated, and the use is non-commercial. See: http://creativecommons.org/licenses/by-nc/4.0/.

\section{REFERENCES}

1. Xie $L$, Kang $H, X u Q$, et al. Sleep drives metabolite clearance from the adult brain. Science 2013;342:373-7.

2. Iliff JJ, Wang M, Zeppenfeld DM, et al. Cerebral arterial pulsation drives paravascular CSF-interstitial fluid exchange in the murine brain. J Neurosci 2013;33:18190-9.

3. lliff JJ, Wang M, Liao Y, et al. A paravascular pathway facilitates CSF flow through the brain parenchyma and the clearance of interstitial solutes, including amyloid $\beta$. Sci Transl Med 2012:4:ra111.

4. Rangroo Thrane V, Thrane AS, Plog BA, et al. Paravascular microcirculation facilitates rapid lipid transport and astrocyte signaling in the brain. Sci Rep 2013;3:2582.

5. Plog BA, Dashnaw ML, Hitomi E, et al. Biomarkers of traumatic injury are transported from brain to blood via the glymphatic system. $J$ Neurosci 2015;35:518-26.

6. Louveau A, Smirnov I, Keyes TJ, et al. Structural and functional features of central nervous system lymphatic vessels. Nature 2015;523:337-41.

7. Pollock $\mathrm{H}$, Hutchings $\mathrm{M}$, Weller $\mathrm{RO}$, et al. Perivascular spaces in the basal ganglia of the human brain: their relationship to lacunes. $J$ Anat 1997;191:337-46.
8. Weller RO, Kida S, Zhang ET. Pathways of fluid drainage from the brain-morphological aspects and immunological significance in rat and man. Brain Pathol 1992;2:277-84.

9. Aspelund A, Antila S, Proulx ST, et al. A dural lymphatic vascular system that drains brain interstitial fluid and macromolecules. $J$ Exp Med 2015;212:991-9.

10. Peng W, Achariyar TM, Li B, et al. Suppression of glymphatic fluid transport in a mouse model of Alzheimer's disease. Neurobiol Dis 2016;93:215-25.

11. Kress BT, lliff JJ, Xia M, et al. Impairment of paravascular clearance pathways in the aging brain. Ann Neurol 2014;76:845-61.

12. Iliff JJ, Chen MJ, Plog BA, et al. Impairment of glymphatic pathway function promotes tau pathology after traumatic brain injury. $J$ Neurosci 2014;34:16180-93.

13. Wang M, Ding F, Deng S, et al. Focal solute trapping and global glymphatic pathway impairment in a murine model of multiple microinfarcts. J Neurosci 2017;37:2870-7.

14. Mestre $\mathrm{H}$, Tithof $\mathrm{J}$, Du T, et al. Flow of cerebrospinal fluid is driven by arterial pulsations and is reduced in hypertension. Nat Commun 2018;9:4878.

15. Goulay R, Flament J, Gauberti M, et al. Subarachnoid hemorrhage severely impairs brain parenchymal cerebrospinal fluid circulation in nonhuman primate. Stroke 2017;48:2301-5.

16. Gaberel T, Gakuba C, Goulay R, et al. Impaired glymphatic perfusion after strokes revealed by contrast-enhanced MRI: a new target for fibrinolysis? Stroke 2014;45:3092-6.

17. Jiang Q, Zhang L, Ding G, et al. Impairment of the glymphatic system after diabetes. J Cereb Blood Flow Metab 2017;37:1326-37.

18. Iliff JJ, Lee H, Yu M, et al. Brain-wide pathway for waste clearance captured by contrast-enhanced MRI. J Clin Invest 2013;123:1299-309.

19. Ratner V, Gao Y, Lee H, et al. Cerebrospinal and interstitial fluid transport via the glymphatic pathway modeled by optimal mass transport. Neuroimage 2017;152:530-7.

20. Ratner V, Zhu L, Kolesov I, et al. Optimal-mass-transfer-based estimation of glymphatic transport in living brain. Proc SPIE Int Soc Opt Eng 2015;9413:9413.

21. Lee $\mathrm{H}$, Xie L, Yu M, et al. The effect of body posture on brain glymphatic transport. J Neurosci 2015;35:11034-44.

22. Davoodi-Bojd E, Ding G, Zhang L, et al. Modeling glymphatic system of the brain using MRI. Neuroimage 2018;188:616-27.

23. Nejad-Davarani SP, Bagher-Ebadian $\mathrm{H}$, Ewing JR, et al. An extended vascular model for less biased estimation of permeability parameters in DCE-T1 images. NMR Biomed 2017;30:e3698.

24. Nejad-Davarani SP, Bagher-Ebadian H, Ewing JR, et al. A parametric model of the brain vascular system for estimation of the arterial input function (AIF) at the tissue level. NMR Biomed 2017;30:e3695.

25. Nedergaard M. Garbage Truck of the Brain. Science 2013;340:1529-30. 